\title{
EARLY DIAGNOSIS OF SEPSIS THROUGH SEPSIS MARKERS AND SEPSIS INDEX VIA FLOW CYTOMETRY TECHNOLOGY
}

\author{
SIMA CHAUHAN ${ }^{1}$, JAGADISH HANSA ${ }^{2 *}$ \\ ${ }^{1}$ Department of Pathology, Institute of Medical Sciences \& SUM Hospital, Siksha O Anusandhan University, Bhubaneswar - 751003, \\ Odisha, India. ${ }^{2}$ Directorate of Medical Research, Institute of Medical Sciences \& SUM Hospital, Siksha 0 Anusandhan University, \\ Bhubaneswar - 751 003, Odisha, India. Email: jagadish.hansa@gmail.com
}

Received: 12 June 2017, Revised and Accepted: 20 July 2017

ABSTRACT

Objective: Sepsis is a major medical challenge, and remains a burden by showing minimal symptoms, non-specific manifestation which leads to the high-mortality rate. The major cause of high mortality rate is due to the diagnostic pitfalls. The sepsis mechanism involves immune suppression associated with multiorgan dysfunction, uncontrolled infection, and death. This study aims to use new parameters in diagnosis of sepsis particularly neutrophil CD64 (nCD64), monocyte human leukocyte antigen-DR (mHLA-DR), performed by flow cytometer.

Methods: Available diagnostic tools such as blood culture (gold standard) and other tools are time-consuming which leads to death because the treatment is not commenced promptly due to unavailability of quick and accurate diagnostic procedures. To overcome such instances, there are different specific cell surface markers which are introduced to reveal sepsis at the earliest using flow cytometry technique. The newer technique allows determination of different cellular and functional pathological components of sepsis. Using this technology, a newer treatment modality (granulocyte macrophage colony-stimulating factor) can be used to reverse sepsis associated immune suppression.

Results: The results suggested that the flow cytometric evaluation of nCD64, mHLA-DR expression assay, seems to be promising as comparison to other tools available for diagnosis.

Conclusion: The pilot data suggest that the flow assay, "sepsis index" is a useful assay for diagnosing and discriminating sepsis in adult at intensive care unit settings in comparison to all the available current modalities of sepsis diagnosis. However, there are no other comparable parameters to diagnose early sepsis.

Keywords: Sepsis, Flow cytometry, Sepsis index.

(C) 2017 The Authors. Published by Innovare Academic Sciences Pvt Ltd. This is an open access article under the CC BY license (http://creativecommons. org/licenses/by/4. 0/) DOI: http://dx.doi.org/10.22159/ajpcr.2017.v10i11.20640

\section{INTRODUCTION}

Sepsis is the leading cause of mortality and morbidity by infection due to multiorgan failure [1-5]. Suspected cases are treated with costly antibiotics, which are often inappropriate and leads to emergence of antibiotic resistant pathogens [6]. Treatment of all suspected cases leads to excessive, costly, and often inappropriate use of antibiotics which can lead to development of antimicrobial resistant pathogens. There is a general agreement that the diagnostic tools available to clinicians are inadequate to rule out sepsis. Definitive diagnosis of sepsis requires (a) clinical identification of infection in a patient who also meets the clinical criteria for the systemic inflammatory response syndrome (SIRS) (diagnosed if patients exhibit at least two of the following readily measurable signs of systemic inflammation (i) altered body temperature $\left[<36^{\circ} \mathrm{C}\right.$ or $>38^{\circ} \mathrm{C}$ ], (ii) increased heart rate [tachycardia; pulse $>90 /$ min], (iii) abnormal white cell count $\left[>12 \times 10^{9} / \mathrm{L}\right.$ or $\left.<4 \times 10^{9} / \mathrm{L}\right]$, and (iv) increased respiratory rate [tachypnoea; respiratory rate $>20$ / min or $\mathrm{PaCO}_{2}>4.2 \mathrm{kPa}$ ]) [7], (b) laboratory test (presently available modalities are complete blood count, Gram-stain, blood culture and antibiotic sensitivity, urine culture and culture of other body fluids, procalcitonin - levels [rapidly increase in sepsis], lactate [increase when there is organ dysfunction], and blood gases [evaluate $\mathrm{O}_{2}$ level and acid base balance] [8]. Comprehensive metabolic panel can be done to monitor health of organs (kidney, lungs), electrolyte balance and blood glucose level. Prothombin time, activated partial thromboplastin time, and other clotting tests are done to detect inflammation in body, cerebrospinal fluid analysis, and others done to identify/rule out other complications such as cardiac biomarkers in heart attack [9]), along with (c) non-laboratory test - (to evaluate health of organs and to detect complications - electrocardiogram, X-ray, computed tomography, magnetic resonance imaging ultrasound, etc.) [9].

From all of the above diagnostic criteria's, SIRS lacks specificity to be clinically meaningful. Main problem of SIRS is the discrimination of with or without infection remains a challenge for clinical setting; thus, it misleads the result interpretation of severe inflammatory response to diagnose sepsis. The blood culture is the gold standard for detection of bacteria and fungi in blood among all the entire laboratory tests. The test is diagnostically useful for a number of specific diseases that result from the spread of bacteria through blood, such as infective endocarditis and infective meningitis. It is also requested routinely for patients with pyrexia of unknown origin in the search for a possible infective cause. Unfortunately, in the context of rapidly evolving sepsis, there is a clinically significant time delay of 2-4 days before blood culture results are available. Blood culture can be falsely negative in cases of sepsis. Thus, there is a need for more rapid and more sensitive methods for identification of bacteria in blood. Recently, specific cell surface markers are introduced to study sepsis $[10,11]$. Sustained sepsis-associated immune suppression is associated with uncontrolled infection, multiorgan dysfunction, and death. Many survivors from sepsis die in the later course of sepsis in a state of fulminant failure of cellular immunity $[3,6,12,13]$. A study by Meisel et al. demonstrated that biomarker-guided granulocyte macrophage colony-stimulating factor therapy is effective and safe for restoring monocytic immunocompetence. Single center trial has shown that the monocytic HLA-DR is a good marker for monitoring the severity of temporary immunodepression after traumatic major surgery or sepsis [14]. Flow 
cytometric (FCM) evaluation of neutrophil CD64 (nCD64) expression assay and also monocyte human leukocyte antigen-DR (mHLA-DR) assay, has got high sensitivity and specificity [11]. This study using above all concept tried to hypothesize the ratio of expression of neutrophilic CD64 (nCD64) and expression of mHLA-DR to diagnose sepsis and to guide the management by including pro- and anti-inflammatory markers of sepsis.

\section{METHODS}

\section{Study population}

This prospective study was conducted from January 2015 to January 2017 at Institute of Medical Sciences (IMS) and SUM Hospital, Bhubaneswar, India. We recruited a total of 126 participants of either sex with suspicion of sepsis as well as 32 healthy controls (blood donors) from the blood bank of the institution. Samples were collected from both groups and analyzed as per the protocol. The clinical criteria of suspicion of sepsis were body temperature instability, cardiovascular instability, and respiratory instability. The participant's blood samples used for this study were obtained from blood drawn for routine laboratory screening procedures as well as for culture. On clinical suspicion of sepsis, blood culture was also performed by blood agar media (Biomericx) and classified the study in two broad classes, i.e., culture positive and culture negative [15]. In routine laboratory screening of participants, the blood was analyzed for total leukocyte count, differential count, platelet count, serum protein, serum electrolytes $\left(\mathrm{NA}^{+}, \mathrm{K}^{+}, \mathrm{Ca}^{2+}\right)$, and glucose. In addition to these other radiological investigations including chest and abdominal X-rays were analyzed. The study was approved by the Institutional Ethical Committee of IMS and SUM Hospital, and as suggested, informed consent by vernacular language was obtained from the legally acceptable representatives.

\section{FCM analysis}

Sample processing and acquisition: Antibodies used in this study were CD14 FITC, CD64 PE CD45 PerCP, and HLA-DR APC. Sample collected was peripheral blood in ethylenediaminetetraacetic acid vial. All the reagents for FCM, analysis were procured from BD Biosciences (San, Jose CA). Protocol used was Stain-Lyse-wash method. $50 \mu \mathrm{l}$ of whole blood was taken in FACS tube to which antibodies were added as per the panel. It was incubated in dark at RT for 15 minutes followed by lyse process using $2 \mathrm{ml}$ "BD FACSLyse" $(\times 1)$. It was followed by two steps of washing using sheath fluid. The cells were resuspended in $0.5 \mathrm{ml}$ of sheath fluid or phosphate-buffered saline. Sample prepared was run on a pre-calibrated flow cytometer. Calibration of instrument was done using the PE QUANTIBRITE BEADS. Acquisition was done with a threshold set on CD 45 PerCP to include lymphocytes, monocytes, and neutrophils. Stop gate used was 30,000 all events or at least 500 monocytes.

\section{Analysis}

A region is drawn on monocytes on an SSC/CD14 plot. Then, "nonmonocytes" was gated on an SSC/CD45 bivariate dot plot and regions drawn on lymphocytes and neutrophils. Median fluorescence intensity of nCD64 and mHLA-DR were recorded. It was then converted to antibodies per cell and sepsis index (SI) was calculated. The protocol for FCM analysis was followed from the protocol of Pradhan et al., 2015 [16].

\section{SI}

The derivation of SI was based on the concept to combine the changes in the expression of pro- and anti-inflammatory markers, and it was calculated from the composite parameter by arithmetic division of antibodies per cell of nCD64 and their respective monocytic HLA-DR and multiplying the ratio by 100 .

$\mathrm{SI}=(\mathrm{nCD} 64 / \mathrm{mHLA}-\mathrm{DR}) \times 100$

Where, nCD64: Neutrophilic CD64 (antibodies per cell), mHLA-DR: Monocytic HLA-DR (antibodies per cell).

\section{Statistical analysis}

All statistical analysis was performed on SPSS version 20 (IBM Corporation). The diagnosis "positive" and "negative" was selected as per the cutoff value range formed from the $90^{\text {th }}$ percentile of controls in case of up regulation and $10^{\text {th }}$ percentile of controls in case of down regulated parameter. Sensitivity and specificity to detect sepsis was calculated based on these cutoffs value range. All the SI data are correlated (Pearson correlation coefficient) with the clinical criteria of sepsis and blood culture findings taken together. Group differences were tested for significance with the Friedman test, and if these results were positive, the non-parametric statistical hypothesis of Wilcoxon test was performed for paired samples.

\section{RESULTS}

The study recruited 126 participants from intensive care unit (ICU)and 32 healthy control groups from the blood bank of the institution. Due to incomplete data, 11 participants were withdrawn from the study; accordingly, 115 cases were analyzed in Table 1.

The nCD64 and mHLA-DR expression were analyzed through FCM parameters and SI. The expression of nCD64 was significantly higher in comparison to healthy control; similarly, expression of mHLA-DR was significantly lower than counterpart of healthy controls (Fig. 1a-c).

Accordingly, SI ranges of infected participants with respect to the SI range of healthy participants were determined in Table 2. All the participants were subclassified into two groups, i.e., infected $(n=75)$ and non-infected $(n=45)$. Further to check the sensitivity and specificity the infected participants were enrolled for culture test.

\section{DISCUSSION}

Early diagnosis of sepsis is important to make crucial decision of therapeutic administration of antimicrobials in a strategic and time bound manner. Several biomarkers found to diagnose the sepsis, namely, C-reactive protein (CRP), procalcitonin, interleukin-6, soluble triggering expressed on myeloid-1, lipopolysacharide-binding protein [17], soluble urokinase-type plasminogen activator receptor, and cell surface markers. According to specificity and sensitivity, there is no such biomarker or biomolecular technique which is currently available and allows a rapid and reliable discrimination between SIRS without infection and sepsis. Each individual marker has some strength as well some limitations. Furthermore, the available markers seem to identify mainly invasive bacterial infection, but parasite, fungi, and viruses may also evoke sepsis. This study examined the "diagnosis of sepsis" through the combination of nCD64 and mHLA-DR (innate immunity markers) to guide clinicians to prescribe the specified treatment to improve the prognosis of mortality rate of sepsis participants. Thus, this study can aid to shorten the decision making process of clinicians and initiation of therapy.

Advanced technique such as flow cytometry is undeniably the best tools for analyzing cell-cell interaction, signaling process, surface markers, proliferation and differentiation, protein secreted by cell, and intracellular molecules [18]. In this study, the cell surface marker nCD64 and mHLA-DR are used to find out the SI with respect to the cut off range of healthy controls.

nCD64 expression is effective in diagnosing sepsis, but due to population heterozygocity, case classification criteria, and assay methodologies; its diagnostic efficacy of sensitivity and specificity varied from 26$97 \%$ and $71-100 \%$, respectively [19]. Median of the intensified fluorescence considered as the parameter of fluorescence intensity is typically a skewed data. The diagnosis "positive" and "negative" was selected as per the cutoff value range formed from the $90^{\text {th }}$ percentile of controls in case of up regulation and $10^{\text {th }}$ percentile of controls in case of downregulated parameter. Sensitivity and specificity to detect sepsis was calculated basing on these cutoff value ranges. This study corroborates that the upregulation of nCD64 of the host's immune 

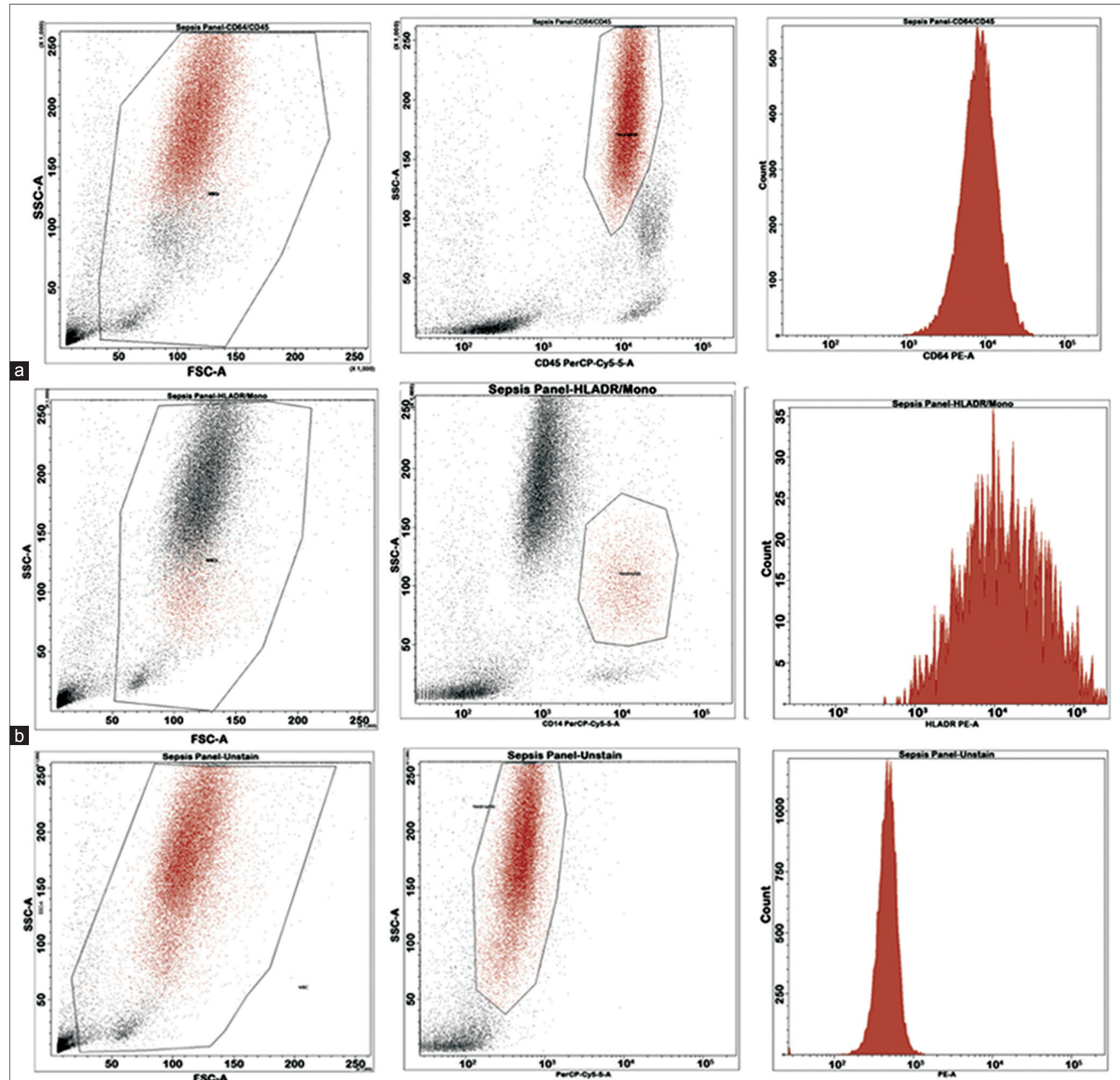

c

Fig. 1: (a) Sepsis panel of CD64/CD45, (b) sepsis panel of human leukocyte antigen-DR/mono, (c) sepsis panel of unstained

Table 1: Represents all the clinical parameters of participants and healthy control group

\begin{tabular}{llll}
\hline S.No. & Parameter & Participants (n=115) & Healthy (n=32) \\
\hline 1 & Male/female & $75 / 40$ & $23 / 9$ \\
2 & Duration of stay in ICU (days) & Average 13 (5-28) & Not applicable \\
3 & Antimicrobial therapy duration (days) & Average $9(7-15)$ & Not applicable \\
4 & CRP $(\mathrm{mg} / \mathrm{L})$ & Average $8.97(3.21-21.23)$ & 3.21 \\
5 & TLC $\left(10^{3} / \mu \mathrm{L}\right)$ & Average $11.12(8.21-20.23)$ & Average $11.98(8.19-13.23)$ \\
6 & I/T ratio & Average $0.21(0.05-0.22)$ & Not done \\
7 & Glucose $(\mathrm{mg} / \mathrm{dL})$ & Average $83(65-105)$ & Not done \\
8 & Procalcitonin $(\mathrm{ng} / \mathrm{ml})$ & Average $4(2-6)$ & Average $0.13(0.11-0.14)$ \\
\hline
\end{tabular}

Data are represented as the median value of the data range. ICU: Intensive care unit, TLC: Total leukocyte count, CRP: C-reactive protein

response to bacterial infection considered being a very early step it is considerably enhanced after $1 \mathrm{hr}$ of invasion. In addition to the nCD64 expression, mHLA-DR down regulation in sepsis seems to be earlier predictive diagnostic marker [20,21].
The mechanism of pro- and anti-inflammatory responses reflects the progression of sepsis, a balanced phenomenon, which can be an effective approach to monitor sepsis [22]. Downregulation of mHLA-DR in antiinflammatory response is relatively rapid and more representative of 
Table 2: SI range of participants and healthy control group

\begin{tabular}{lll}
\hline Parameters & Healthy control & MICU-sepsis \\
\hline Cases & 32 & 115 \\
nCD64 (Ab/cell) & $268-1045$ & $1250-7835$ \\
mHLA-DR (Ab/cell) & $16052-48106$ & $1296-15025$ \\
SI & $0.93-5.23$ & $17.41-332.5$ \\
\hline
\end{tabular}

SI=(nCD64/mHLA-DR) $\times 100$. nCD4: Neutrophil CD64, mHLA-DR: Monocyte

human leukocyte antigen-DR, SI: Sepsis index, MICU: Medical intensive care unit

physiological changes [21] whereas the pro-inflammatory markers such as CRP and nCD64 typically have a prolonged rise following pathogenic challenge [23,24]. Accordingly, "SI" a composite parameter was derived in combination with mHLA-DR and nCD64, which increased the diagnostic sensitivity, reiterating the importance of using both parameters (Table 2).

Our study corroborates various FCM methods which can predict prognosis in sepsis [25-27] increased mortality in adults is being associated with upregulation of nCD64 $[28,29]$ while downregulation showed for non-survivors [30]; however, in sepsis, nCD64 showed a prognostic significance [25]. In another study, Ng et al. demonstrated no significant difference between infected and non-infected or control groups for mHLA-DR expression [31]. In our study, a trend analysis of mHLA-DR indicated that mHLA-DR was significantly downregulated in non-survivors only at a later stage.

\section{CONCLUSION}

The pilot data suggest that the flow assay "SI" appears to be useful for diagnosing and discriminating sepsis in adult at ICU settings in comparison to all other available current modalities for sepsis diagnosis. FCM evaluation of nCD64, mHLA-DR expression assay is very affirmative.

\section{ACKNOWLEDGMENT}

All authors thankful to Dr. Paresh Jain, George Bainik and Dr. Sumanta Basu, BD Biosciences, Pabitra Mohan Mohanty and Manoranjan Mohapatra staff of SUM Hospital for their technical help. Special thanks to Somadatta Das, Central Research Laboratory of this institute for his contribution to frame the image for the manuscript. There is no financial support for this study

\section{REFERENCES}

1. Angus DC, Linde-Zwirble WT, Lidicker J, Clermont G, Carcillo J, Pinsky MR. Epidemiology of severe sepsis in the United States: Analysis of incidence, outcome, and associated costs of care. Crit Care Med 2001;29(7):1303-10.

2. Angus DC, Pereira CA, Silva E. Epidemiology of severe sepsis around the world. Endocr Metab Immune Disord Drug Targets 2006;6(2):207-12.

3. Annane D, Bellissant E, Cavaillon JM. Septic shock. Lancet 2005;365(9453):63-78

4. Annane D, Aegerter P, Jars-Guincestre MC, Guidet B; CUB-Réa Network. Current epidemiology of septic shock: The CUB-Réa Network. Am J Respir Crit Care Med 2003;168(2):165-72.

5. RussellJA. Management of sepsis. NEnglJMed2006;355(16):1699-713.

6. Hotchkiss RS, Karl IE. The pathophysiology and treatment of sepsis. N Engl J Med 2003;348(2):138-50.

7. Levy MM, Fink MP, Marshall JC, Abraham E, Angus D, Cook D, et al. $2001 \mathrm{SCCM} / \mathrm{ESICM} / \mathrm{ACCP} / \mathrm{ATS} / \mathrm{SIS}$ international sepsis definitions conference. Crit Care Med 2003;31(4):1250-6.

8. Turki AK, Sulaiman SA. Eleveated blood pressure among patients with hypertension in general hospital of Penang, Malaysia: Does poor adherence matter? Int J Pharm Pharm Sci 2010;2(1):24-32.

9. Swarnakar K, Vagha J. Sepsis biomarkers in early onset neonatal sepsis-a review. Internet J Infect Dis 2013;11(1):1-9.

10. Lehr HA, Krombach F, Münzing S, Bodlaj R, Glaubitt SI, Seiffge D, et al. In vitro effects of oxidized low density lipoprotein on CD11b/ CD18 and L-selectin presentation on neutrophils and monocytes with relevance for the in vivo situation. Am J Pathol 1995;146(1):218-27.

11. Ng PC, Li G, Chui KM, Chu WC, Li K, Wong RP, et al. Neutrophil CD64 is a sensitive diagnostic marker for early-onset neonatal infection. Pediatr Res 2004;56(5):796-803.

12. Cohen J. The immunopathogenesis of sepsis. Nature 2002;420(6917):885-91.

13. Hoesel LM, Gao H, Ward PA. New insights into cellular mechanisms during sepsis. Immunol Res 2006;34(2):133-41.

14. Meisel C. Granulocyte-macrophage colony stimulating factor to reverse sepsis-associated immunosuppression-a double blind, randomized, placebo-controlled multicenter trial. Am J Respir Crit Care Med 2009; 180:640-8.

15. Devraj B, Kali A, Charles MV, Seetha KS. Modified biphasic media for blood culture. Asian J Pharm Clin Res 2016;9(3):42-3.

16. Pradhan R, Jain P, Paria A, Saha A, Sahoo J, Sen A, et al. Ratio of neutrophilic CD64 and monocytic HLA-DR: A novel parameter in diagnosis and prognostication of neonatal sepsis. Cytometry B 2015;64:1-8.

17. Simms HH, D'Amico R. Lipopolysaccharide induces intracytoplasmic migration of the polymorphonuclear leukocyte $\mathrm{CD} 11 \mathrm{~b} / \mathrm{CD} 18$ receptor. Shock 1995;3(3):196-203.

18. Shyam KB, Bhat KI. Apoptosis and flow cytometric studies of Bauhinia variegata bark extract. Asian J Pharm Clin Res 2014;7(1):45-7.

19. Hoffmann JJ. Neutrophil CD64 as a sepsis biomarker. Biochem Med (Zagreb) 2011;21(3):282-90.

20. Birle A, Nebe CT, Gessler P. Age-related low expression of HLADR molecules on monocytes of term and preterm newborns with and without signs of infection. J Perinatol 2003;23(4):294-9.

21. Azizia M, Lloyd J, Allen M, Klein N, Peebles D. Immune status in very preterm neonates. Pediatrics 2012;129(4):e967-74.

22. Venet F, Lepape A, Monneret G. Clinical review: Flow cytometry perspectives in the ICU-from diagnosis of infection to monitoring of injury-induced immune dysfunctions. Crit Care 2011;15(5):231.

23. Barth E, Fischer G, Schneider EM, Wollmeyer J, Georgieff M, Weiss M. Differences in the expression of CD64 and mCD14 on polymorphonuclear cells and on monocytes in patients with septic shock. Cytokine 2001;14(5):299-302.

24. Haseena SB, Bai MM, Pallavi C, Thyagaraju K. Suppression of paracetamol toxicity by antioxidant principles of Hybanthus enneaspermus (L.) F. Muell. In mice blood and liver. Int J Pharm Pharm Sci 2011;3(4):90-4.

25. Motta M, Zini A, Regazzoli A, Garzoli E, Chirico G, Caimi L, et al. Diagnostic accuracy and prognostic value of the CD64 index in very low birth weight neonates as a marker of early-onset sepsis. Scand J Infect Dis 2014:46(6):433-9.

26. Monneret G, Lepape A, Voirin N, Bohé J, Venet F, Debard AL, et al. Persisting low monocyte human leukocyte antigen-DR expression predicts mortality in septic shock. Intensive Care Med 2006;32(8):117583.

27. Park SH, Park BG, Park CJ, Kim S, Kim DH, Jang S, et al. An extended leukocyte differential count (16 types of circulating leukocytes) using the cyto diff flow cytometric system can provide informations for the discrimination of sepsis severity and prediction of outcome in sepsis patients. Cytometry B Clin Cytometry 2014;86:244-56.

28. Livaditi O, Kotanidou A, Psarra A, Dimopoulou I, Sotiropoulou C, Augustatou K, et al. Neutrophil CD64 expression and serum IL-8: Sensitive early markers of severity and outcome in sepsis. Cytokine 2006;36(5-6):283-90.

29. Song SH, Kim HK, Park MH, Cho HI. Neutrophil CD64 expression is associated with severity and prognosis of disseminated intravascular coagulation. Thromb Res 2008;121(4):499-507.

30. Danikas DD, Karakantza M, Theodorou GL, Sakellaropoulos GC, Gogos CA. Prognostic value of phagocytic activity of neutrophils and monocytes in sepsis. Correlation to CD64 and CD14 antigen expression. Clin Exp Immunol 2008;154(1):87-97.

31. Ng PC, Li G, Chui KM, Chu WC, Li K, Wong RP, et al. Quantitative measurement of monocyte HLA-DR expression in the identification of early-onset neonatal infection. Biol Neonate 2006;89(2):75-81. 